\title{
Effects of Infrared Energy on Dual Elliptical NDIR Ethanol Gas Sensors ${ }^{\dagger}$
}

\author{
Jinho Kim, Sangho Shin and Seunghwan Yi * \\ Dept. of Mechanical Eng., Korea National University of Transportation (KNUT), 50 Daehakro, Chungjushi, \\ Chungbuk 27469, Korea; wlsgh0614@naver.com (J.K.); kagnew@naver.com (S.S.) \\ * Correspondence: isaac_yi@ut.ac.kr; Tel.: +82-42-841-5129 \\ + Presented at the Eurosensors 2017 Conference, Paris, France, 3-6 September 2017.
}

Published: 5 September 2017

\begin{abstract}
This paper presents the effects of infrared energies on dual elliptical nondispersive infrared (NDIR) ethanol gas sensors for preventing drunken drivers and the advantages of using dual ethanol detectors for temperature compensation algorithm. In order to achieve long-term reliability according to the aging of infrared source, two ethanol detectors are placed at the two foci of ellipsoids and the averaged voltage ratios of two ethanol detectors are used to establish the compensation methods.
\end{abstract}

Keywords: nondispersive infrared gas sensor; ethanol gas sensor; dual-ethanol detectors; elliptical structure; energy density; temperature compensation

\section{Introduction}

The NDIR ethanol gas sensor and Near-IR ethanol detecting system are currently researching and developing as promising candidates for driver alcohol detection system for safety (DADSS) [1]. Among the alternatives, the former was recognized as a more competitive one than the latter because of the size, the cost and compatibility to the electrochemical sensor type which is currently using in automotive and industrial areas as a screening device. Due to the above reasons, a unique NDIR ethanol gas sensor has been invented and its properties were briefly presented in terms of its temperature dependent outputs and also compensation methods to predict the concentrations of ethanol gas irrelevant to the changes of ambient temperatures [2]. However, reliable sensing methods were needed to overcome the long-term stability that would be raised by the aging of infrared source. So, this paper tried to devise a new method by using two identical elliptical structures and ethanol detectors.

\section{Simulations and Experiments}

\subsection{Simulations and Fabrication}

Figure 1 shows the simulation results of irradiated energies at the active thermopile areas of dual-ethanol sensors. When there is no hindrance of the irradiation during the transmission of infrared light, the infrared light is focused as a small circular spot at the active area of thermopile detector as shown in Figure 1a. However, when the mirror surfaces are partially blocked (about 6 $\mathrm{cm}$ ), the received energy at the active area of thermopile is diminished about $40 \%$ with the same structure and showed dispersed beam pattern within the active area of thermopile detector as can be seen in Figure 1b. Because the voltage of thermopile detector is proportional to the infrared energy of source as described by Beer-Lambert law in Equation (1),

$$
I_{d}(T, x)=I_{o}(T) \cdot \exp [-\beta(T) \cdot x],
$$


where, $I_{o}(T)$ is the initial energy that is radiated from the source, $\beta(T)$ is the product of the gas absorption coefficient and optical path length, $x$ is the concentration of target gas.

As described in Figure 1, the energy densities at the detectors are different in both cases [3]; however, the optical path lengths and the absorption coefficients of ethanol gas are identical in this structure. Then, the output voltage ratio would be independent on the concentrations of ethanol gas, however, it will be changed with the temperature variations.
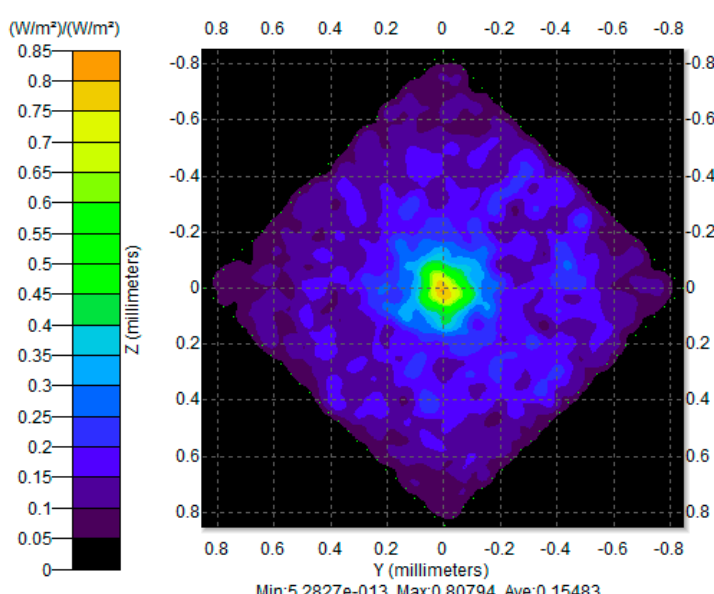

Min:5.2827e-013, Max:0.80794, Ave:0.15483
Total Flux:0.046179 W, Flux Emitted Flux:0.076965, 8167 Incident Rays

(a)
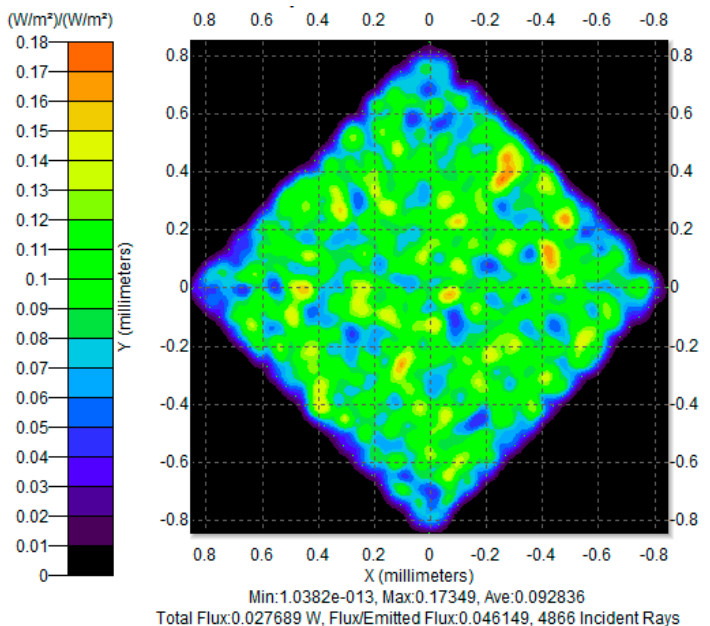

(b)

Figure 1. Simulation results of the irradiated infrared energies at the active areas of thermopile detector: (a) full elliptical structure, (b) partially blocked $(6 \mathrm{~cm})$ elliptical structure.

Based upon the above simulation results, dual elliptical waveguide for NDIR ethanol gas sensors were 3-D modeled and fabricated as can be seen in Figure 2 [2,3]. In this sensor module, two ethanol detectors are positioned at each focal point of dual elliptical structures for ethanol gas sensing and achieving the output voltages as a function of ethanol concentrations and ambient temperatures with one infrared source and two identical optical path lengths [3].

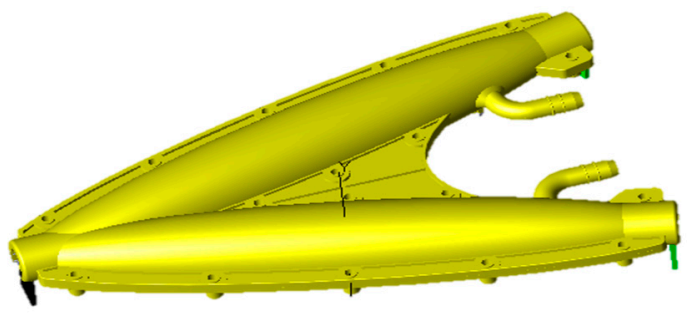

(a)

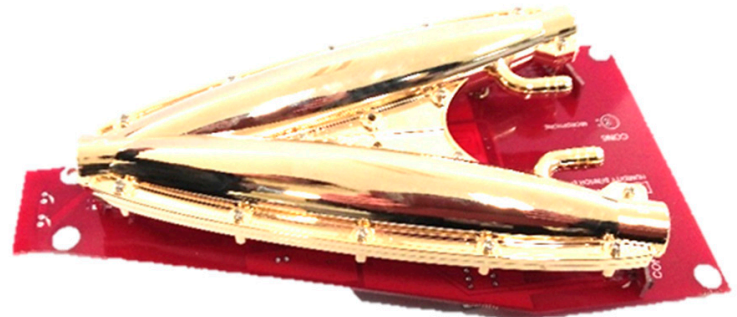

(b)

Figure 2. Dual-elliptical structure for ethanol gas sensors: (a) 3-D modelling structure, (b) fabricated dual-elliptical ethanol gas sensor.

\subsection{Experiments}

After implementing the basic algorithm into the microcontroller unit, the prototyped ethanol gas sensor was installed in the gas measurement system and the testing for the characterization of ethanol gas sensor has been conducted as described in previous article [3]. 


\section{Results and Discussion}

The output voltages of ethanol sensor (at the full elliptical structure) are presented in Figure 3 as a function of ethanol concentrations and ambient temperatures from $253 \mathrm{~K}$ to $333 \mathrm{~K}$. They are regressed as exponential equation as presented in Equation (2),

$$
V_{d F}(T, x)=V_{o F}(T) \cdot \exp [-\beta(T) \cdot x],
$$

where, $V_{O F}(T)$ is the output voltage of ethanol gas sensor at $0 \mathrm{ppm}$. Even though the initial output voltages at zero ethanol concentration were dependent on the structures (full and partially blocked elliptical structures), the output voltages of ethanol detectors followed the Equation (2).

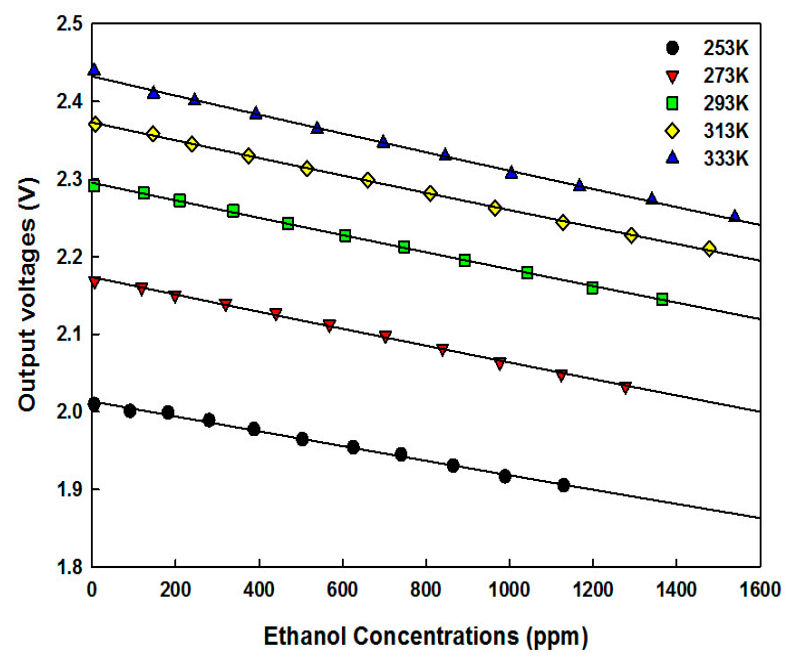

Figure 3. Output voltages of ethanol gas sensor as a function of ethanol gas concentrations from $253 \mathrm{~K}$ to $333 \mathrm{~K}$ (with full elliptical structure).

After downloading the output voltages from the dual ethanol detectors, the ratios of output voltages have been calculated and presented in Figure 4. As can be seen in Figure 4, the ratios of output voltages of ethanol detectors showed almost constant value at the measured temperature and they could be formulated by 3rd-order of polynomial according to the ambient temperatures. The ratio of output voltages was independent of the variations of ethanol gas concentrations.

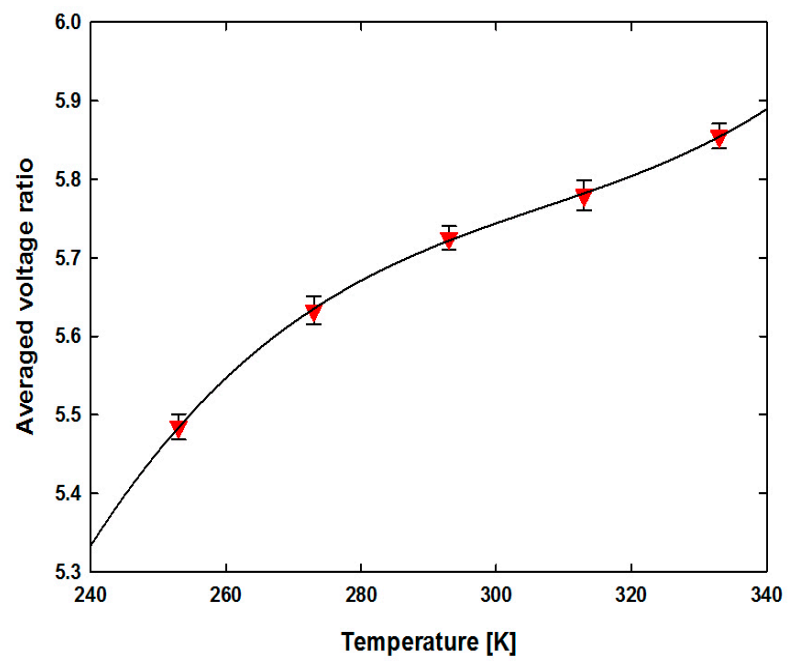

Figure 4. Averaged ratios of output voltages of dual-elliptical ethanol gas sensors ranging from $253 \mathrm{~K}$ to $333 \mathrm{~K}$ temperatures. 


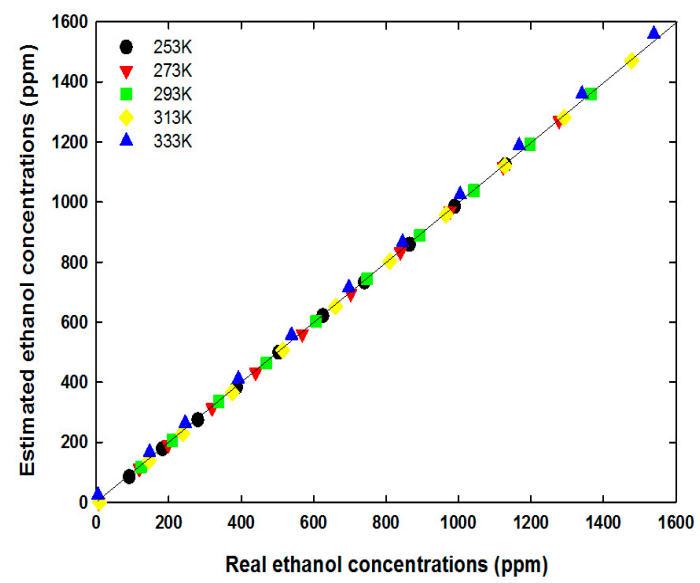

Figure 5. Estimated ethanol concentrations as a function of ethanol concentrations and ambient temperatures after temperature and offset calibration.

After implementing the temperature compensation algorithm [4] and offset values according to the variations of ambient temperatures, the estimated ethanol concentrations could be presented as in Figure 5. Due to the differences of initial values between the measurements and the regressions, the errors below $100 \mathrm{ppm}$ ethanol gas were little high around $\pm 15 \%$. However, above $100 \mathrm{ppm}$ ethanol concentrations, the range of average estimation errors was between $-1.2 \%$ to $3.0 \%$ from $253 \mathrm{~K}$ to $333 \mathrm{~K}$ temperature variations and within the tested ethanol concentrations.

\section{Conclusions}

NDIR ethanol gas sensor has been successfully fabricated with two identical ethanol detectors and elliptical waveguide structures: partially blocked and intact elliptical shapes. The ratios of output voltages of ethanol detectors were almost constant within the measured ethanol concentrations. From the theoretical approaches, the estimation of ethanol concentrations could be accurately achieved by using the ratios of output voltages and the difference of gas absorption coefficients. The long-term stability could be enhanced by using this method because the ratio of output voltages might be independent on the infrared energy due to the aging of infrared source.

Acknowledgments: This research was supported by R \& D Center for Green Patrol Technologies through the R \& D for Global Top Environmental Technologies funded by Ministry of Environment, Republic of Korea (MOE) and the authors would like to thank Ja-Young Lee for the summaries of experimental data.

Conflicts of Interest: The authors declare no conflict of interest and the founding sponsors had no role in the design of the study; in the collection, analyses, or interpretation of data; in the writing of the manuscript, and in the decision to publish the results.

\section{References}

1. Zaouk, A.; Will, M.; Traube, E.; Strassburger, R. Driver Alcohol Detection System for Safety (DADSS)-A Status update. In Proceedings of the 24 Enhanced Safety Vehicles, Gotenburg, Sweden, 10 June 2015.

2. Kim, J.H.; Lee, K.H.; Yi, S.H. NDIR ethanol gas sensor with two elliptical optical structures. Procedia Eng. 2016, 168, 359-362, doi:10.1016/j.proeng.2016.11.122.

3. Kim, J.H.; Yi, S.H. Properties of Non-dispersive infrared Ethanol Gas Sensors according to the Irradiation Energy. J. Sens. Sci. Tech. 2017, 26, 168-172, doi:10.5369/JSST.2017.26.3.168.

4. Yi, S.H. Temperature compensation methods of nondispersive infrared $\mathrm{CO}_{2}$ sensor with dual elliptical waveguide. Sens. Mater. 2017, 29, 243-252, doi:10.18494/SAM.2017.1439.

(C) 2017 by the authors. Licensee MDPI, Basel, Switzerland. This article is an open access article distributed under the terms and conditions of the Creative Commons Attribution (CC BY) license (http://creativecommons.org/licenses/by/4.0/). 